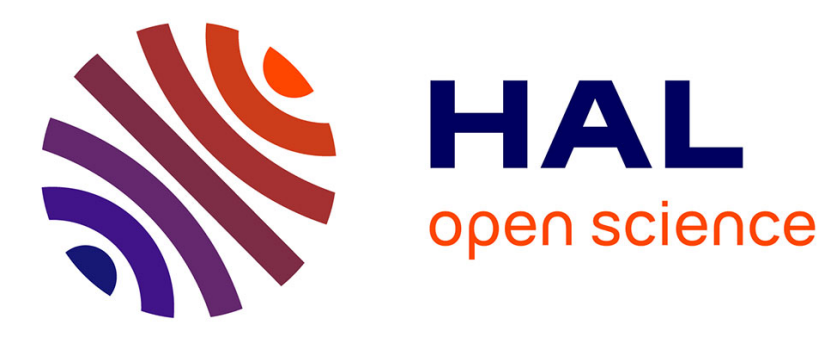

\title{
Influence of flank face on the condition of chatter self-excitation during turning
}

Alexander M. Gouskov, Mikhail Guskov, Philippe Lorong, Grigory Panovko

\section{To cite this version:}

Alexander M. Gouskov, Mikhail Guskov, Philippe Lorong, Grigory Panovko. Influence of flank face on the condition of chatter self-excitation during turning. International Journal of Machining and Machinability of Materials, 2017, 19 (1), pp.17. 10.1504/ijmmm.2017.081186 . hal-02733403

\section{HAL Id: hal-02733403 \\ https://hal.science/hal-02733403}

Submitted on 2 Jun 2020

HAL is a multi-disciplinary open access archive for the deposit and dissemination of scientific research documents, whether they are published or not. The documents may come from teaching and research institutions in France or abroad, or from public or private research centers.
L'archive ouverte pluridisciplinaire $\mathbf{H A L}$, est destinée au dépôt et à la diffusion de documents scientifiques de niveau recherche, publiés ou non, émanant des établissements d'enseignement et de recherche français ou étrangers, des laboratoires publics ou privés. 


\title{
INFLUENCE OF FLANK FACE ON THE CONDITION OF CHATTER SELF-EXCITATION DURING TURNING
}

\author{
Alexandre GOUSKOV \\ Applied Mechanics Department, \\ Bauman Moscow State Technical University; Moscow, Russia; \\ Fax: +7 4992674844 \\ E-mail: gouskov_am@mail.ru
}

\section{Mikhail GUSKOV* Philippe LORONG}

PIMM Laboratory,

CNRS, Arts \& Metiers ParisTech, CNAM

Paris, France

Fax: +33144246382

E-mail: mikhail.guskov@ensam.eu

E-mail: philippe.lorong@ensam.eu

Corresponding author

\section{Grigoriy PANOVKO}

Vibrations Laboratory,

RAS Institute of Machine Science

Moscow, Russia

Fax: +7 4956249863

E-mail: gpanovko@yandex.ru

\begin{abstract}
This paper addresses a modelling approach for cutting tool and workpiece material interaction with ploughing taken into account. In the proposed model the distributed contact on the flank face is replaced by a concentrated additional cutting edge characterised by two physical parameters and bringing about a new delay term into the dynamical system. It is shown that under monoharmonic excitation of the tool on the direction orthogonal to the cutting velocity, it is possible to isolate the effect of the flank face and observe experimentally its parameters. The influence of the ploughing on the instability boundaries of continuous cutting is investigated. An analytical expression is obtained for the effective linear damping coefficient due to ploughing and depending on the cutting velocity.
\end{abstract}

Keywords: continuous cutting stability; nonlinear dynamics; tool-workpiece interaction; cutting stiffness; flank face; ploughing; chatter; turning; PoincaréAndronov-Hopf bifurcation. 
Reference to this paper should be made as follows: Gouskov, A., Guskov, M., Lorong, P., Panovko, G. 'Influence of the Flank Face on the Condition of Chatter Self-Excitation During Cutting During Turning', Int. J. Machining and Machinability of Materials, Vol. $\mathrm{x}$, No. x, pp.xxx-xxx.

Biographical notes: Alexander M. Gouskov graduated from Bauman Moscow State Technical University (BMSTU) in 1972, he received a PhD in 1977 and the degree of Doctor of Sciences in 1997. He is actually full Professor in BMSTU, his field of interest covering Nonlinear Dynamics, Theory of Stability, Chaotic Systems, Dynamics of Technological Systems, Numerical Methods.

Mikhail Guskov graduated from Bauman Moscow State Technical University (BMSTU) in 2003 and received a $\mathrm{PhD}$ in 2007 in the field of Nonlinear Rotordynamics. He is associate professor at the Ecole Nationale Supérieure d'Arts et Métiers (ENSAM, France) since 2009. His field of interest is Nonlinear Dynamics, Machining Dynamics, Rotordynamics, Structural Health Monitoring.

Philippe Lorong is Professor of Mechanical Engineering at the Ecole Nationale Supérieure d'Arts et Métiers (ENSAM, France) since 2008. He received a teaching degree (agrégation, 1987) and a $\mathrm{PhD}$ on a Parallel Approach for Structural Analysis, Numerical behaviour on Concurrent Computers (1993) and a Habilitation (2006) on the contribution of structural analysis on numerical modelling of machining at the macroscopic and mesoscopic scales.

Grigory Panovko (Dr. of Sc., Professor) graduated from Bauman Moscow State Technical University (BMSTU) in 1970. He is working in Blagonravov Institute of Machines Sciences of Russian Academy of Sciences (IMASH RAS) as Head of Vibromechanics Laboratory and also Professor in the BMSTU Department of Applied Mechanics, Member of IFToMM, Member of the Russian Academy of Engineering. Prof. Panovko is author of over 150 technical paper and 16 patents. His research interests include modeling of dynamics of mechanical systems.

\section{Introduction}

Various approaches have been proposed for modeling the ploughing phenomena during the history of the cutting process investigation. The historical qualitative description of ploughing is given by Tobias (1965). Mainly, the following phenomena accompanying the ploughing are observed: increase of friction forces, flank wear, increase of cutting forces, unstable domains reduction, especially for low cutting velocities, etc. As for the macroscopic dynamics, most works elaborate one of the two following approaches: either a friction term, linear or nonlinear, is introduced as a function of the relative normal velocity of the cutting edge w.r.t. the processed surface (Fofana et al. (2003)), or a force term proportional to the volume swept by the tool's flank face (Enders et al. (1995); Lee et al. (1995); Moradi et al. (2010)). These phenomena result in apparent damping, proposed by Tobias (1965), also referred to as process damping (Altintas (2000)). A more detailed description of ploughing force, based on the flank face-workpiece interference volume, has been presented by $\mathrm{Wu}(1989)$. This approach has then been applied to various processes, such as drilling (Guibert and Paris (2008)) or milling (Mehdi and Zghal (2012)). In practical applications, the ploughing is sometimes modelled directly by a drastic increase in damping (Seguy et al. (2008)). 


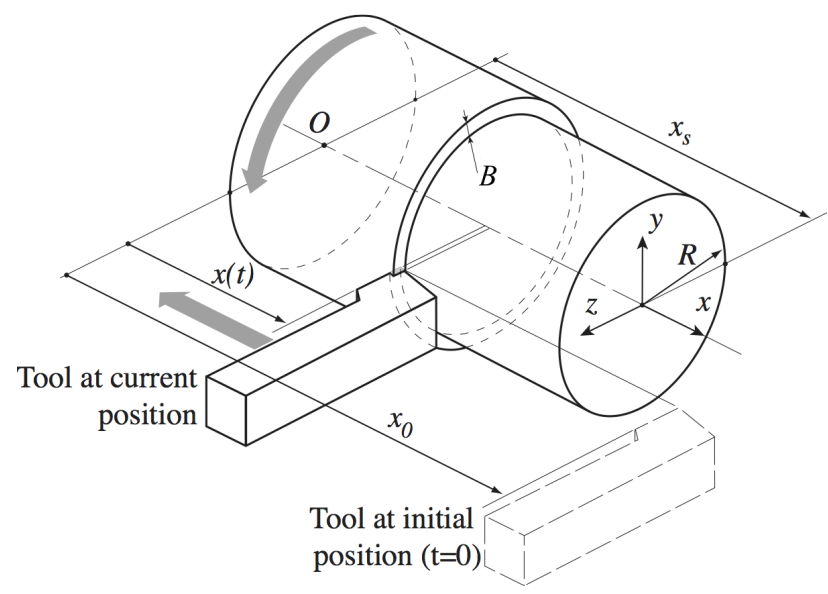

Figure 1 Schematic of the turning operation

By the following, the mechanical aspect of the interaction of the tool's flank face with the machined surface during straight turning (fig. 1, fig. 2 left), under orthogonal cutting conditions in $x$-y plane (the tool is supposed stiff in $z$-direction and relatively compliant in $x$-direction). Thus only the $z$-oriented cutting edge-related kinematics are considered along the $x$-axis. Under turning, a complex process of tool-workpiece interaction and of chip formation is observed (fig. 2 left). A wide review of the state of the art in modelling and investigation of turning dynamics and stability is provided by Siddhpura and Paurobali (2012).

It is also noticed that the interaction effects on the flank face during steady cutting are impossible to single out and in the overall behaviour and is an unobservable parameter. It is although pointed out that with the decrease of the cutting speed, the effects of the ploughing phenomena influence sensibly the chatter onset and can lead to an important change in the energy dissipation (Tarng et al (1994); Lee et al. (1995)) .

Recently, an alternative, mechanistic-type approach has been developed, based on the representation of the flank face interaction via a fictitious auxiliary cutting edge, following the main edge closely (Paris et al. (2008); Bondarenko et al. (2009); Bondarenko (2010)). These works had shown the relevance of auxiliary edge model in case of drilling and serve as basis to the developments of the present paper. The goal of the present work is to provide a representation of the flank face interaction via the secondary cutting edge concept in case of turning, to validate its relevance w.r.t. ploughing and to investigate its impact on the dynamic stability of quasi-steady turning.

We first begin, in section 2, by introducing our ploughing model based on the usage of an auxiliary cutting edge. Definition of the surface generation, including the new cutting edge, is also given in this the section. We then detail, in section 3, the tool-workpiece interaction model. The proposed model is elaborated in order to mix, in a consistent way, cutting and ploughing contributions into the total cutting force. The effect of the auxiliary cutting edge for monoharmonic axial vibrations of the tool at a multiple of the workpiece rotation frequency is studied in section 4 . It exhibits the contribution of the auxiliary cutting edge on the cutting force work induced by the vibrations. At last, in section 5, dynamics of vibration cutting is considered. A comparison with 'classical' approaches of process damping is proposed. 


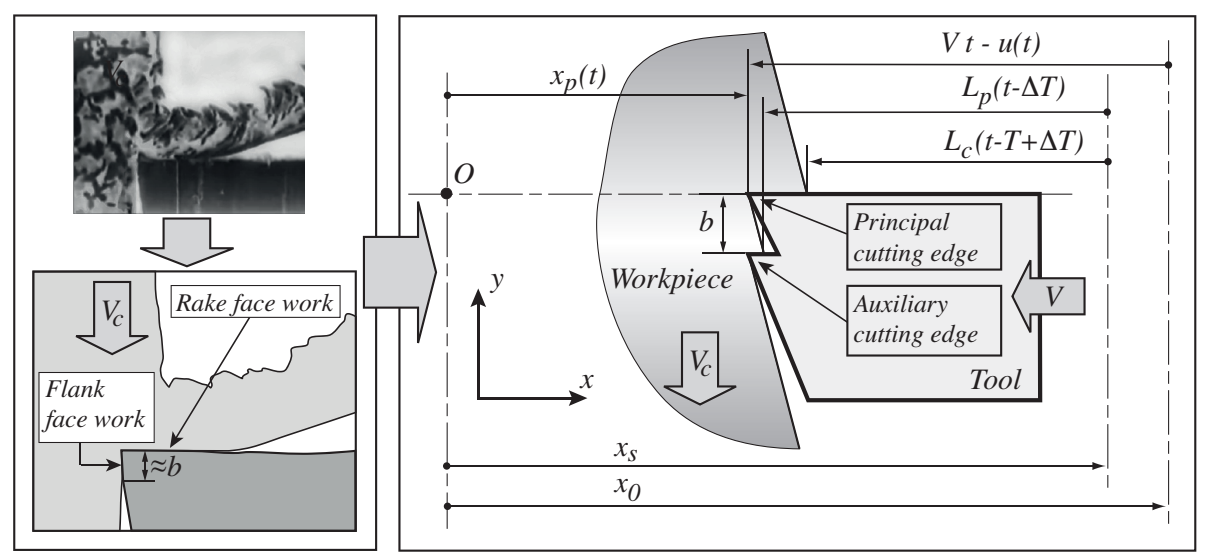

Figure 2 Schematic of the cutting part of the tool with the auxiliary cutting edge on the flank face; $b$ flank face effective width, $V_{c}$ cutting speed, $V$ feed rate

\section{Ploughing modelling description}

\section{Auxiliary cutting edge.}

The interaction of the flank face with the processed material is manifested by additional forces and by change in resulting surfaces geometry (material's peening). To model the interaction on the flank face, we propose to replace the whole flank face by one auxiliary cutting edge (Bondarenko et al. (2009); Bondarenko (2010)), situated at distance $b$ from the principal cutting edge (fig. 2). This interaction on flank face is reduced to a cutting process with coefficient (this concept will be precised in the following) $K_{c 0}$. The introduction of two parameters $-\left\{b, K_{c 0}\right\}$-allows one to consider observable (measurable) cutting forces caused by both the cutting itself and ploughing, from one common viewpoint. The parameters related to the principal cutting edge will be marked with the subscript $p$, those related to the auxiliary edge - with the subscript $c$.

\section{Equations of new surfaces generation.}

Geometrical equations for the new surfaces generated by the principal and the auxiliary cutting edges during the cylindrical surface turning (or drilling) (Gouskov et al. (2001)) are written as follows:

$$
\begin{aligned}
x_{p}(t) & =x_{0}-V t+u(t), & & x_{c}(t)=x_{0}-V t+u(t), \\
D_{p}(t) & =x_{s}-L_{c}(t-T+\Delta T)-x_{p}(t), & & D_{c}(t)=x_{s}-L_{p}(t-\Delta T)-x_{c}(t), \\
h_{p}(t) & =\max \left(0, D_{p}(t)\right), & & h_{c}(t)=\max \left(0, D_{c}(t)\right), \\
L_{p}(t) & =L_{c}(t-T+\Delta T)+h_{p}(t), & & L_{c}(t)=L_{p}(t-\Delta T)+h_{c}(t), \\
L_{p}(t & \leq 0) \equiv 0, & & L_{c}(t \leq 0) \equiv 0,
\end{aligned}
$$

with $x_{p}$ - coordinate of the principal cutting edge, measured from the spindle, along its axis; $x_{0}$ - initial position of the cutting edge; $x_{s}$ - coordinate of the part's free end; $u-$ axial displacement of the cutting edge with respect to its nominal position, caused by the deformation of the tool support assembly; $D_{p}$ - distance from the cutting edge at instant $t$ to 
the surface generated by the auxiliary cutting edge at instant $t-(T-\Delta T), T-$ workpiece rotation period, $\Delta T$ - time corresponding to the rotation at angle $b / R, b$-distance between the principal and the auxiliary edges along the direction of $V_{c} ; R$-machined surface radius; $h_{p}$ - principal cutting edge's cutting thickness; $L_{p}$ - coordinate of the surface generated by the principal cutting edge, measured from the free end $\left\{x_{0} \geq x_{s}\right\}$. Analogous parameters for the auxiliary cutting edge are denoted by the $c$ subscript $-\left\{x_{c}, D_{c}, h_{c}, L_{c}\right\}$. The kinematical relations (1) allow one to compute the coordinates of the surfaces generated by turning $L_{p}(t), L_{c}(t)$, as well as the uncut thicknesses of the removed material $h_{p}(t), h_{c}(t)$ and the position of the cutting edges $x_{p}(t), x_{c}(t)$ for a given motion of the tool $u(t)$, with initial conditions: $x_{0}, x_{s}, L_{p}(t \leq 0), L_{c}(t \leq 0)$, defined on the initial set.The variables $D_{p}(t)$, $D_{c}(t)$ are auxiliary, and allowing to account for discontinuous cutting.

The feed per turn is $a=V T$, with $V$ - axial feed rate. The delay between the principal and the auxiliary cutting edge $\Delta T$ is computed as follows:

$$
T=\frac{1}{f}, \quad C=2 \pi R, \quad \beta=\frac{b}{C}, \quad \Delta T=\beta T,
$$

with $f$-workpiece rotation frequency. The time interval between the passage of the auxiliary and the principal cutting edges is $T-\Delta T=T(1-\beta)$.

In case when only the principal cutting edge is engaged (cutting without ploughing $\left.h_{c} \equiv 0 \Rightarrow L_{c}(t)=L_{p}(t-\Delta T)\right)$ the system of equations (1) reduces to

$$
\begin{aligned}
& x_{p}(t)=x_{0}-V t+u(t), \\
& D_{p}(t)=x_{s}-L_{p}(t-T)-x_{p}(t), \\
& h_{p}(t)=\max \left(0, D_{p}(t)\right), \\
& L_{p}(t)=L_{p}(t-T)+h_{p}(t), \\
& L_{p}(t \leq 0) \equiv 0 .
\end{aligned}
$$

\section{Two types of continuous cutting.}

- Continuous cutting with the principal edge alone $-h_{c} \equiv 0, h_{p}>0 \forall t$.

In this case, the equation system (2) applies. $D_{p}$ is always positive, $D_{p}>0 \forall t$ and can be eliminated from the writing because $h_{p}(t) \equiv D_{p}(t)$ :

$$
L_{p}(t)=x_{s}-x_{p}(t), \quad h_{p}(t)=V T-(u(t)-u(t-T)) .
$$

For quasistatic cutting $(u(t)=$ const $)$ the uncut chip thickness reads

$$
h_{p}(t)=V T=a .
$$

- Continuous cutting with both edges $-h_{c}>0, h_{p}>0 \forall t$.

In this case one has to consider the whole system (1), while $D_{p}, D_{c}$ can again be eliminated:

$$
\begin{array}{ll}
L_{p}(t)=x_{s}-x_{p}(t), & h_{p}(t)=V(T-\Delta T)-(u(t)-u(t-(T-\Delta T))), \\
L_{c}(t)=x_{s}-x_{c}(t), & h_{c}(t)=V \Delta T-(u(t)-u(t-\Delta T)) .
\end{array}
$$

For quasistatic cutting $(u(t)=$ const $)$ the uncut chip thicknesses read

$$
h_{p}(t)=a(1-\beta), \quad h_{c}(t)=a \beta .
$$

The equations (3)-(5) are also valid for the of absolutely rigid tool case $(u \equiv 0)$. 


\section{Tool-workpiece interaction model}

The tool-workpiece interaction model defines the relation between the cutting force $P$ and the uncut chip thickness $h$ and is typically determined experimentally in quasistatic conditions $(u(t)=$ const) under assumption of unique cutting part. In the present study it is only necessary to deal with the component of the cutting force in the direction of vibration, ie direction $x$, fig. 2 . The cutting force $P$ is thus here a scalar.

The uncut chip thickness in our case equals the feed per turn $a$. In these conditions, the contributions of the cutting force (principal edge) and of ploughing (auxiliary edge) are impossible to distinguish. It would be of interest to the experimenter to represent the cutting force model in such a manner that the quasistatic force has expression does not depend whether the ploughing is present or not. In the following, the fractional-rational model proposed in Bondarenko (2010); Brissaud et al. (2007); Paris et al. (2008) will be used. The experimental data is processed using the similarity theory framework (Gouskov (2013)). Under assumption of unique cutting edge (one point cutting model - 1PC), the resulting cutting force $P(t)$, can be written as follows:

$$
P(t)=K_{0} H \frac{\frac{h(t)}{H}+r \frac{h(t)^{2}}{H^{2}}}{1+\frac{h(t)}{H}} .
$$

with $\left\{K_{0}, H, r\right\}$ - experimental coefficients, $h(t)$ - chip thickness, units:

$$
\left[K_{0}\right]=\mathrm{N} \cdot \mathrm{m}^{-1}, \quad[H]=\mathrm{m}, \quad[r]=1, \quad[h]=\mathrm{m} .
$$

The parameter $H$ serves as a characteristic length scale for the interaction in a given toolworkpiece pair (Brissaud et al. (2007)).

The ploughing force's contribution can be accounted for via an auxiliary cutting edge, as proposed in Bondarenko et al. (2009). Under assumption of two cutting edges (two point cutting model $-2 \mathrm{PC}$ ), the resulting cutting force $P(t)$ is a sum of contributions due to the principal cutting edge $-P_{p}(t)$, and to the auxiliary one $-P_{c}(t)$ :

$$
\begin{aligned}
P(t) & =P_{p}(t)+P_{c}(t), \\
P_{p}(t) & =K_{p 0} H_{p} \frac{\frac{h_{p}(t)}{H_{p}}+r_{p} \frac{h_{p}(t)^{2}}{H_{p}^{2}}}{1+\frac{h_{p}(t)}{H_{p}}}, \quad P_{c}(t)=K_{c 0} h_{c}(t),
\end{aligned}
$$

with $\left\{K_{p 0}, H_{p}, r_{p}, K_{c 0}\right\}$ - experimental coefficients; $\left\{h_{p}(t), h_{c}(t)\right\}$ - uncut chip thickness for the principal and auxiliary edges. In general, the parameters $\left\{h_{p}(t), h_{c}(t)\right\}$ are defined by the equations (1).

Static stiffness coefficients for each model ( $K$ for $1 \mathrm{PC} ; K_{p}, K_{c}$ for 2PC), are fundamental for the stability evaluation in case of continuous cut. These coefficients define the slope of the thickness-force function:

$$
\begin{array}{rlrl}
K & =\frac{\partial P}{\partial h}=K_{0} \kappa(h / H ; r), & \kappa(h / H ; r)=r+\frac{1-r}{(1+h / H)^{2}} \\
K_{p}=\frac{\partial P}{\partial h_{p}}=K_{p 0} \kappa_{p}\left(h_{p} / H_{p} ; r_{p}\right), & \kappa_{p}\left(h_{p} / H_{p} ; r_{p}\right)=r_{p}+\frac{1-r_{p}}{\left(1+h_{p} / H_{p}\right)^{2}}, \\
K_{c}=\frac{\partial P}{\partial h_{c}}=K_{c 0} . &
\end{array}
$$


For given material, tool and chosen cut depth $B$ (chip width in the $z$ direction, see fig. 1 , given the orthogonal cutting conditions) the quantities $\left\{K_{0}, K_{p 0}, K_{c 0}, r, r_{p}\right\}$ (9) remain constant. Also, the normalized stiffness coefficient $\left\{\kappa, \kappa_{p}\right\}$, depending on the normalised cut thicknesses $\left\{h / H, h_{p} / H_{p}\right\}$ and dimensionless parameters $\left\{r, r_{p}\right\}$, varyin finite ranges

$$
r \leq \kappa \leq 1, \quad r_{p} \leq \kappa_{p} \leq 1
$$

The coefficients $\left\{K_{0}, K_{p 0}, K_{c 0}\right\}$, equal to the cut stiffness when the uncut chip thickness tends to zero (see equation (9): $h, h_{p} \rightarrow 0$ ), for a given cut depth $B$. It is important to mention that while $K_{0}$ and $K_{p 0}$ can be obtained experimentally by classical approaches, the auxiliary edge's parameter $K_{c 0}$ would require specific tests, involving vibrations in the feed direction at multiples of the workpiece rotation frequency, as pointed out by Bondarenko et al. (2009). According to dimensional analysis, these coefficients would depend on a characteristic stress quantity for the workpiece material (such as yield limit, ultimate strength, elasticity modulus) $\sigma_{L}$ in the following way (Brissaud et al. (2007)):

$$
K_{0}=\gamma \sigma_{L} B, \quad K_{p 0}=\gamma_{p} \sigma_{L p} B, \quad K_{c 0}=\gamma_{c} \sigma_{L c} B
$$

here $\left\{\gamma, \gamma_{p}, \gamma_{c}\right\}$ are dimensionless coefficients, depending mainly on the cutting edges' shape, sharpening, coatings, etc. The nature of these coefficients for the two edges can be substantially different because of the respective physical phenomena at stake in cutting and ploughing.

During the quasistatic cutting, the conditions (4), (6) are verified and, after substitution into (7), (8) we obtain the following equation

$$
K_{0} H \frac{\frac{a}{H}+r \frac{a^{2}}{H^{2}}}{1+\frac{a}{H}}=K_{p 0} H_{p} \frac{\frac{a(1-\beta)}{H_{p}}+r_{p} \frac{a^{2}(1-\beta)^{2}}{H_{p}^{2}}}{1+\frac{a(1-\beta)}{H_{p}}}+K_{c 0} a \beta .
$$

By balancing the terms with equal powers of $a$, we obtain the relations connecting the parameters $\left\{K_{0}, H, r\right\}$ with $\left\{K_{p 0}, H_{p}, r_{p}, K_{c 0}, \beta\right\}$ :

$$
\begin{aligned}
& K_{0}=K_{p 0}(1-\beta)+K_{c 0} \beta, \\
& \frac{K_{0} r}{H}=\frac{K_{p 0} r_{p}}{H_{p}}(1-\beta)^{2}+\frac{K_{c 0}}{H_{p}} \beta(1-\beta), \\
& \frac{1}{H}=\frac{1-\beta}{H_{p}} .
\end{aligned}
$$

It is of practical interest to represent the auxiliary edge-related cut stiffness as $K_{c 0}$ via a factor w.r.t. the principal edge cut stiffness $K_{p 0}$

$$
K_{c 0}=\psi K_{p 0} .
$$

It should be noticed that the flank face stiffness is substantially higher than that of the principal face $-\psi>1$. The solution of (11) in terms of the principal edge's parameters $\left\{K_{0}, H, r\right\} \mapsto\left\{K_{p 0}, H_{p}, r_{p}\right\}$ reads as follows:

$$
\begin{array}{lll}
K_{p 0}=\frac{K_{0}}{1-\beta+\beta \psi} & \Rightarrow & K_{0}=K_{p 0}(1-\beta+\beta \psi), \\
r_{p}=\frac{r(1-\beta+\beta \psi)-\beta \psi}{1-\beta} & \Rightarrow & r=\frac{r_{p}(1-\beta)+\beta \psi}{1-\beta+\beta \psi}, \\
H_{p}=H(1-\beta) & \Rightarrow H=\frac{H_{p}}{1-\beta} .
\end{array}
$$


By adding the auxiliary cutting edge, instead of three "observed" parameters, $\left\{K_{0}, H, r\right\}$, we now consider five $-\left\{K_{p 0}, H_{p}, r_{p}, \beta, \psi\right\}$. Three of these parameters, namely $\left\{K_{p 0}, H_{p}, r_{p}\right\}$ are determined univocally via $\left\{K_{0}, H, r\right\}$ and via two internal parameters related to the auxiliary cutting edge $-\{\beta, \psi\}$ (12). The relation for the case $\beta=0$ (without considering two edges), according to the tool-workpiece interaction model, (8) corresponds to (7) and the kinematical equations (1) reduce to (2). I.e., the internal parameters $\{\beta, \psi\}$, accounting for ploughing, cannot be determined from quasistatic cutting experiments and only qualitative analysis is possible in this context.

\section{Monoharmonic axial vibrations of the tool}

Consider continuous cutting involving both cutting edges $h_{c} \geq 0, h_{p} \geq 0, \forall t$ with superimposed axial monoharmonic vibration of the tool with the oscillation period $T_{e}$ :

$$
u(t)=e \sin (\varphi(t)), \quad \varphi(t)=\frac{2 \pi}{T_{e}} t,
$$

with $e$ vibration magnitude. Suppose the workpiece rotation period is a multiple of that of the tool oscillation: $T=T_{e} n, n=1,2,3, \cdots$. This case is interesting because when only the principal edge is engaged, the uncut chip thickness is constant $h(t)=h_{p}(t)=$ const and thus the cutting force would be constant also (3). However, when both edges are involved - principal and auxiliary,- thicknesses cut by each edge $\left\{h_{p}(t), h_{c}(t)\right\}$ vary periodically, according to (5), and can be expressed in the following way:

$$
h_{p}(t)=a(1-\beta)-\Delta u_{p}(t), \quad h_{c}(t)=a \beta-\Delta u_{c}(t),
$$

with

$$
\begin{aligned}
& \Delta u_{p}(t)=u(t)-u(t-T(1-\beta))=e(1-\cos B) \sin (\varphi(t))-e \sin B \cos (\varphi(t)), \\
& \Delta u_{c}(t)=u(t)-u(t-T \beta)=e(1-\cos B) \sin (\varphi(t))+e \sin B \cos (\varphi(t)),
\end{aligned}
$$

with $B=2 \pi n \beta$. The condition for continuous cutting writes (from second relation in (13) and the expression of $\Delta u_{c}(t)$ in (14)):

$$
e \leq \frac{1}{2} a \beta
$$

Since the distance between the cutting edges is substantially smaller than the turned surface radius, $\beta<<1$, the principal edge uncut chip thickness will have a small periodic component $\max \left|\Delta u_{p}\right| \leq a(1-\beta)$. The cutting force (8) can be written as a sum of a constant and periodic parts, $P_{0}$ and $\Delta P(t)$ respectively:

$$
P(t)=P_{0}+\Delta P(t),
$$

or, more specifically,

$$
\begin{aligned}
& P_{0}=K_{0} H \frac{\alpha+r \alpha^{2}}{1+\alpha} \\
& \Delta P(t) \simeq-K_{p 0} \kappa_{p}\left(\alpha, r_{p}\right) \Delta u_{p}(t)-K_{c 0} \Delta u_{c}(t) .
\end{aligned}
$$


Here $\alpha=a / H-$ is the feed per turn, divided by a characteristic distance scale of the cutting process model (7). This expression for $\Delta P$ is a simplification where the cross terms of $\Delta u_{p}$ and $\Delta u_{c}$ are neglected. The periodic part of the cutting force $\Delta P(t)=\Delta P\left(t+T_{e}\right)$ includes an inphase $\Delta P_{S} \sin (\varphi(t))$ and quadrature $\Delta P_{C} \cos (\varphi(t))$ components.

The quadrature component realizes a nonzero work over a period of tool oscillation

$$
\Delta W_{T_{e}}=\int_{t=0}^{t=T_{e}} \Delta P(t) d u(t)=-\pi K_{p 0} e^{2}\left(\psi-\kappa_{p}\left(\alpha, r_{p}\right)\right) \sin B .
$$

The stiffness of the auxiliary cutting edge is substantially higher than the principal one, i.e. as $\kappa_{p}\left(\alpha, r_{p}\right)<1$ and $\psi>1$ we have $\psi>\kappa_{p}\left(\alpha, r_{p}\right)$, and for those relative frequencies $n$, for which $\sin B>0, B=2 \pi n \beta$ the average work of the cutting forces turns out to be negative (16). This leads to energy dissipation caused by the flank face work. At the same time, if $\sin (2 \pi n \beta)<0$, which is possible if $(j-1 / 2) / \beta<n<j / \beta, j=1,2, \cdots$, then, according to (16), the cutting force would perform positive work $\Delta W_{T_{e}}>0$. Thus, in spite of the intuitive behavior this force can serve as a source of excitation of vibrations of a machining system. For example, when $b=0.02$, positive work is possible in the frequency range $25<n<50$. It could be a possible explanation for vibration auto-excitation in machining systems working at small cutting velocities and high relative frequencies oscillation (it should be noticed that when $\psi<\kappa_{p}\left(\alpha, r_{p}\right)$, ploughing might cause oscillation at high cutting velocities also).

In general, for random ratio between the period of axial vibration $T_{e}$ and the workpiece rotation period $T$, the work of cutting forces over one oscillation period is computed as follows:

$$
\Delta W_{T_{e}}=-\pi K_{p 0} e^{2}\left[\psi \sin (2 \pi n \beta)+\kappa_{p}\left(\alpha, r_{p}\right) \sin (2 \pi n(1-\beta))\right], \quad n=\frac{T}{T_{e}}
$$

If in (17) one has $\beta=0$, then

$$
\Delta W_{T_{e}}=-\pi K_{p 0} e^{2} \kappa\left(\alpha, r_{p}\right) \sin (2 \pi n),
$$

and it appears that for the case of only one cutting edge, there exist frequency ratio values, for which the cutting process is a source of energy to the system $\Delta W_{t_{e}}>0$, and the frequency of vibrations is not a multiple to the rotation frequency $\sin (2 \pi n)<0$. It is important to notice that for integer $n$, in absence of flank face interaction, the power of cutting forces is a constant value and the vibrational component (18) would be zero.

It is practical to represent the numerical results in a universal, dimensionless framework. For scale factors of time, distance, and force, $\left\{T_{*}, L_{*}, P_{*}\right\}$ we take

$$
T_{*}=T, \quad L_{*}=H, \quad P_{*}=K_{0} H .
$$

Then we can introduce the following dimensionless quantities:

dimensionless forces

$$
\Pi=\frac{P}{P_{*}}, \quad \Pi_{p}=\frac{P_{p}}{P_{*}}, \quad \Pi_{c}=\frac{P_{c}}{P_{*}},
$$


average over one oscillation

period

$$
\Pi_{0}=\frac{1}{T_{e}} \int_{0}^{T_{e}} \Pi(t) d t, \quad \Pi_{p 0}=\frac{1}{T_{e}} \int_{0}^{T_{e}} \Pi_{p}(t) d t, \quad \Pi_{c 0}=\frac{1}{T_{e}} \int_{0}^{T_{e}} \Pi_{c}(t) d t
$$

variation over one oscillation period

$$
\Delta \Pi=\Pi-\Pi_{0}, \quad \Delta \Pi_{p}=\Pi_{p}-\Pi_{p 0}, \quad \Delta \Pi_{c}=\Pi_{c}-\Pi_{c 0},
$$

dimensionless feed and oscillation magnitude

$$
\alpha=\frac{a}{L_{*}}, \quad \varepsilon=\frac{e}{L_{*}},
$$

dimensionless work of cutting forces over one oscillation period

$$
\Delta \Psi_{T_{e}}=\frac{\Delta W_{T_{e}}}{P_{*} L_{*}}=-\pi \varepsilon^{2} \frac{\psi \sin (2 \pi n \beta)+\kappa_{p}\left(\alpha, r_{p}\right) \sin (2 \pi n(1-\beta))}{1-\beta+\beta \psi}
$$

dimensionless vibratory speed of the cutting part of the tool

$$
\nu=\frac{T_{*}}{L_{*}} \frac{d u}{d t} .
$$

\section{Example of flank face influence on process damping}

On fig. 3 a dimensionless cutting force model according to (7), (8), (12), (15) is shown. The influence of the effective length of the flank face (distance form the principal to the auxiliary cutting edge expressed by $\beta$ ) under axial vibration of the tool at a multiple frequency of that of the workpiece rotation is shown on fig. 4,5 . One can notice the following features:

- the approximate computation based on (15) provides the results that are very close to the full ones (without neglecting cross terms),

- for small relative vibration frequencies $(n=3)$, the ploughing leads to damping $\left(\Delta \Psi_{T_{e}}<0\right)$

- at high vibration frequencies $(n=30)$, on the contrary, the ploughing can induce vibration of the machining system $\left(\Delta \Psi_{T_{e}}>0\right)$.

It can be seen that with an increase in $\beta$ for low relative frequencies of vibrations, the amplitude of cutting forces also increase (fig. 4(a)) and the dissipated energy due to the cutting process also increases. The work of the flank face is the main contribution to the vibratory part of the cutting forces (fig. 5(b) and 5(c)). Analogous data is available for high frequency vibrations $(n=30)$. However, as distinct from the low frequency case the work of the flank face can lead to positive energy input, as determined by the phase relations between the cutting force and the tool vibrations, which is clearly seen in the force-displacement diagram - fig. 5(d). 


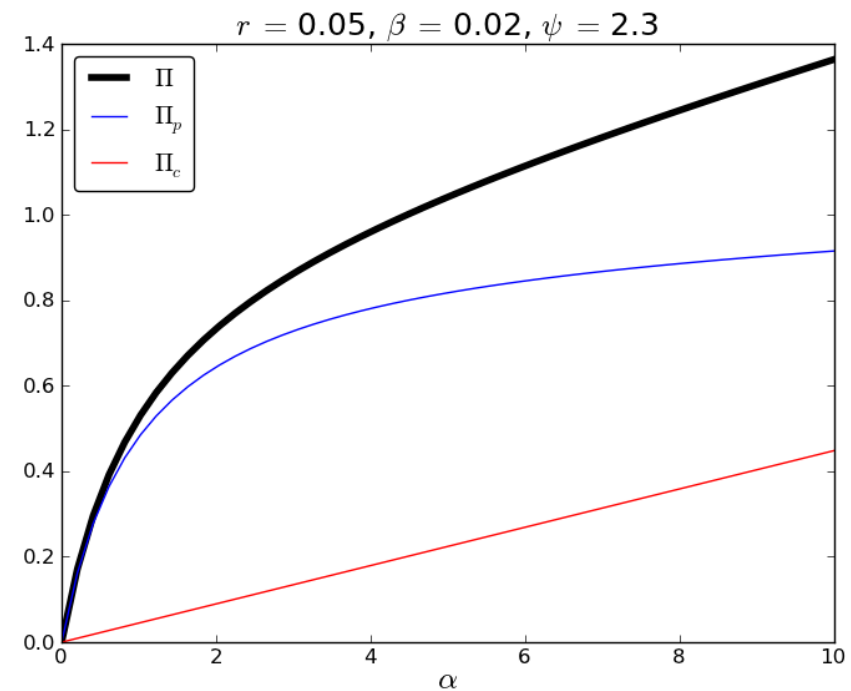

Figure 3 Distribution of the cutting force (black line) between the principal (blue line) and auxiliary (red line) cutting edges.

\section{Dynamics of vibration cutting}

The tool dynamics are considered, under stiff workpiece assumption. The tool is represented by a 1 DOF system, parameterized by $u$-displacement of the tool along the workpiece axis, perpendicular to the cutting velocity, mass $m$, linear stiffness of the mounting $k$ and linear damping coefficient $\zeta$. The tool is subject to the cutting force $P(8)$. Tool vibrations are described by the equation of motion

$$
m \frac{d^{2} u}{d t^{2}}+2 \zeta \sqrt{k m} \frac{d u}{d t}+k u=P .
$$

This model includes surfaces generation relations (1) and cutting force model (8).

Dimensionless representation of the dynamics of vibratory cutting.

The following characteristic scales are chosen: time scale - one revolution duration of the workpiece $T$, [s], distance scale - cutting force model parameter $H,[\mathrm{~m}]$, characteristic stiffness - tool support stiffness $k,\left[\mathrm{~N} . \mathrm{m}^{-1}\right]$, force scale - product $-k H,[\mathrm{~N}]$ :

- dimensionless time

$$
t=T \tau, \quad \frac{d}{d \tau}()=()^{\prime}
$$

- dimensionless forces

$$
\Pi=\frac{P}{k H}, \quad \Pi_{p}=\frac{P_{p}}{k H}, \quad \Pi_{c}=\frac{P_{c}}{k H},
$$




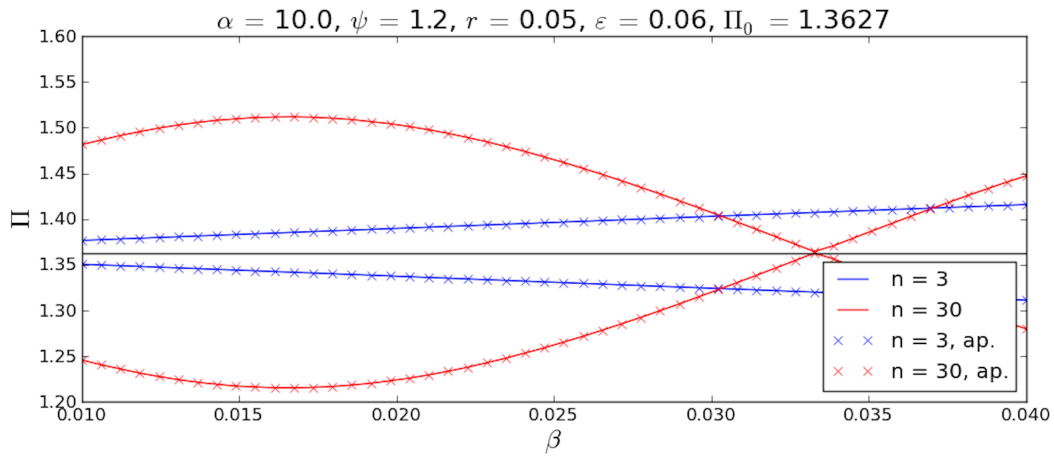

(a) variation of cutting forces

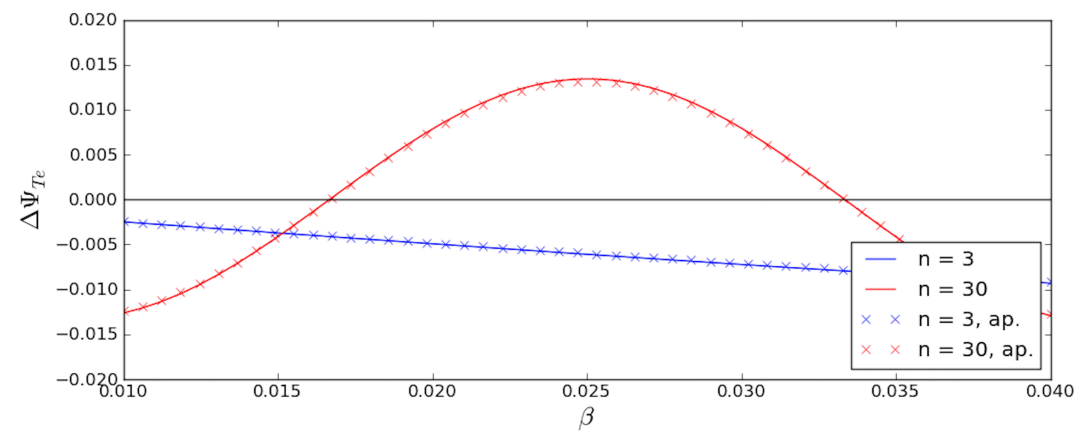

(b) variation of cutting forces' work during one period of oscillations

Figure 4 Influence of the effective flank face length $\beta$ on the characteristics of the cutting for low frequency axial vibration (blue line, $n=3$ ) and for high frequency axial vibration (red line, $n=30$ ), solid line: full computation, cross: approximation (15). 


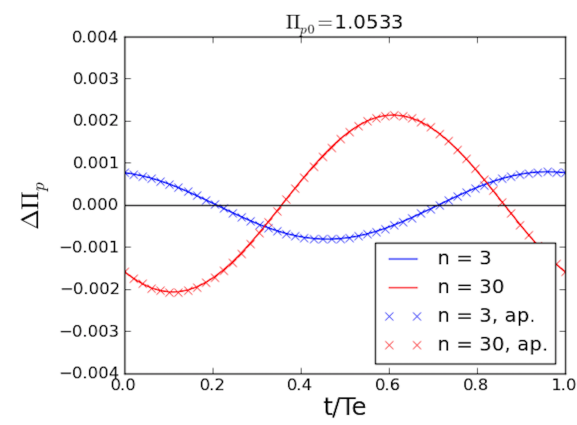

(a) principal edge cutting force variation over one oscillation period

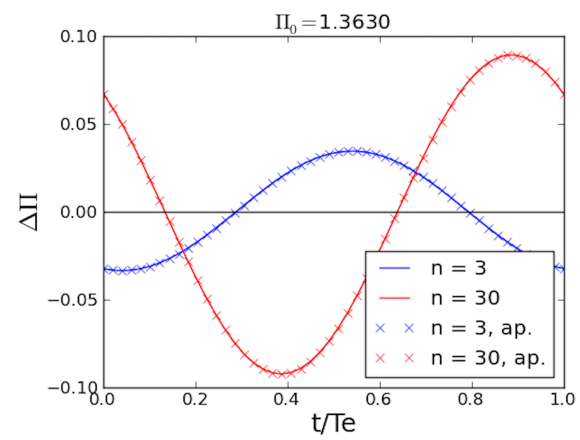

(c) cutting force variation over one oscillation period

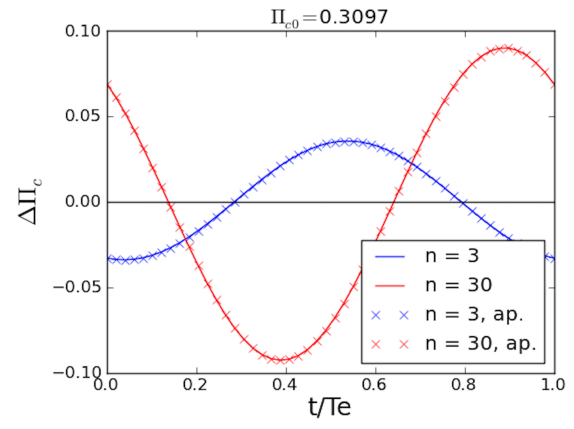

(b) auxiliary edge cutting force variation over one oscillation period

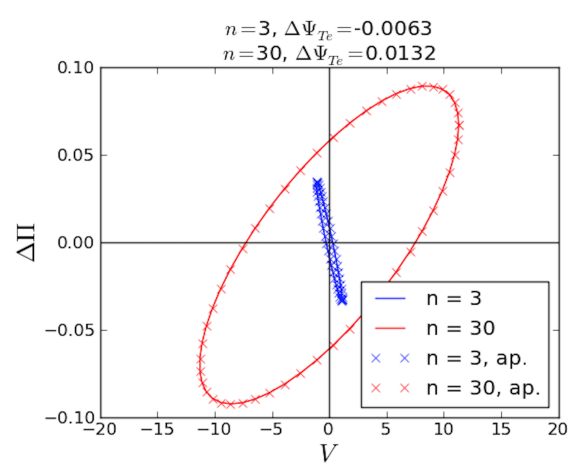

(d) force-displacement diagram

Figure 5 Cutting process characteristics variation for low frequency axial vibration (blue line, $n=3)$ - vibrations lead to energy dissipation $\left(\Delta \Psi_{T_{e}}<0\right)$ and high frequency (red line) - vibration leads to energy input into the system $\left(\Delta \Psi_{T_{e}}>0\right)$. 
- dimensionless stiffnesses

$$
\mathrm{K}=\frac{K}{k}, \quad \mathrm{~K}_{p}=\frac{K_{p}}{k}, \quad \mathrm{~K}_{c}=\frac{K_{c}}{k}, \quad \mathrm{~K}_{0}=\frac{K_{0}}{k},
$$

- dimensionless thicknesses

$$
\mathrm{H}_{p}=\frac{H_{p}}{H}, \quad \alpha=\frac{a}{H},
$$

- dimensionless size parameters

$$
\left\{\mathrm{X}_{p}, \mathrm{X}, \xi, \Lambda_{p}, \Lambda_{c}, \eta, \eta_{p}, \eta_{c}, \Delta_{p}, \Delta_{c}\right\}=\frac{1}{H}\left\{x_{p}, x_{c}, u, L_{p}, L_{c}, h, h_{p}, h_{c}, D_{p}, D_{c}\right\}
$$

We also introduce the following notations

$$
f_{0}=\frac{1}{2 \pi} \sqrt{\frac{k}{m}}, \quad f=\frac{1}{T}, \quad F=\frac{f}{f_{0}}=\frac{V_{c}}{R} \sqrt{\frac{m}{k}},
$$

with $f_{0}$ - tool's eigenfrequency, $f$ - workpiece rotation frequency, $F$ - relative frequency of the workpiece rotation (rotation frequency, devided by the tool's eigenfrequency). The tool dynamics eqation (19) takes the following form:

$$
\frac{F^{2}}{4 \pi^{2}} \xi^{\prime \prime}+2 \zeta \frac{F}{2 \pi} \xi^{\prime}+\xi=\Pi .
$$

The new surface generation relations (1) become

$$
\begin{array}{ll}
\mathrm{X}_{p}(\tau)=\mathrm{X}_{0}-\tau+\xi(\tau), & \mathrm{X}_{c}(\tau)=\mathrm{X}_{0}-\tau+\xi(\tau), \\
\Delta_{p}(\tau)=\Lambda_{c}(\tau-1+\beta)-\mathrm{X}_{p}(\tau), & \Delta_{c}(\tau)=\Lambda_{p}(\tau-\beta)-\mathrm{X}_{c}(\tau), \\
\eta_{p}(\tau)=\max \left(0, \Delta_{p}(\tau)\right), & \eta_{c}(\tau)=\max \left(0, \Delta_{c}(\tau)\right), \\
\Lambda_{p}(\tau)=\Lambda_{c}(\tau-1+\beta)+\eta_{p}(\tau), & \Lambda_{c}(\tau)=\Lambda_{p}(\tau-\beta)+\eta_{c}(\tau), \\
\Lambda_{p}(\tau \leq 0) \equiv 0, & \Lambda_{c}(\tau \leq 0) \equiv 0 .
\end{array}
$$

The cutting model for the principal and auxiliary edges (8) can be written as follows

$$
\Pi(\tau)=\mathrm{K}_{p 0} \mathrm{H}_{p} \frac{\frac{\eta_{p}(\tau)}{\mathrm{H}_{p}}+r_{p} \frac{\eta_{p}(\tau)^{2}}{\mathrm{H}_{p}^{2}}}{1+\frac{\eta_{p}(\tau)}{\mathrm{H}_{p}}}+\mathrm{K}_{c 0} \eta_{c}(\tau),
$$

with

$$
\mathrm{K}_{p 0}=\frac{\mathrm{K}_{0}}{1-\beta+\beta \psi}, \quad \mathrm{K}_{c 0}=\psi \mathrm{K}_{p 0} .
$$

Respective characteristic stiffness coefficients from (9) are computed as follows

$$
\begin{array}{ll}
\mathrm{K}=\frac{\partial \Pi}{\partial \eta}=\mathrm{K}_{0} \kappa(\eta ; r), & \kappa(\eta ; r)=r+\frac{1-r}{(1+\eta)^{2}} \\
\mathbf{K}_{p}=\frac{\partial \Pi}{\partial \eta_{p}}=\mathrm{K}_{p 0} \kappa_{p}\left(\eta_{p} / H_{p} ; r_{p}\right), & \kappa_{p}\left(\eta_{p} / H_{p} ; r_{p}\right)=r_{p}+\frac{1-r_{p}}{\left(1+\eta_{p} / H_{p}\right)^{2}}, \\
\mathbf{K}_{c}=\frac{\partial \Pi}{\partial \eta_{c}}=\mathrm{K}_{c 0} . &
\end{array}
$$


Equations (21), (22), (23) describe dynamics of turning of cylindrical surfaces with ploughing taken into account via two cutting edges (model 2CP). Kinematical relations (22) enable investigations of discontinuous cutting by means of additional variables $\left\{\Lambda_{p}, \Lambda_{c}, \Delta_{p}, \Delta_{c}\right\}$ (20), (22). For the case $\beta=0$ these relations can be applied to the case of absence of ploughing $-\eta_{c} \equiv 0$ and $\eta=\eta_{p}$. In the following the problem of stability of continuous cutting is analyzed to bring forward the influence of the ploughing on the stability boundaries. More specifically, the case of continuous engagement of both edges is considered $\left(\eta_{c}>0, \eta_{p}>0, \forall \tau\right)$. In this case, the equations of new surfaces generation (22) simplify and enable the explicit expression of uncut chip thickness for each edge:

$$
\begin{aligned}
& \eta_{p}(\tau)=\alpha(1-\beta)+\xi(\tau-1+\beta)-\xi(\tau), \\
& \eta_{c}(\tau)=\alpha \beta+\xi(\tau-\beta)-\xi(\tau) .
\end{aligned}
$$

\section{Continuous cutting stability.}

Consider the case of continuous cutting without vibrations $\xi(\tau)=\xi^{0}=$ const. From relations (25) the uncut chip thicknesses read

$$
\eta_{p}^{0}(\tau)=\alpha(1-\beta), \quad \eta_{c}^{0}(\tau)=\alpha \beta
$$

From equations (23) one finds the constant cutting forces:

$$
\Pi_{p}^{0}=\mathrm{K}_{p 0} \mathrm{H}_{p} \frac{\alpha+r_{p} \alpha^{2}}{1+\alpha}, \quad \Pi_{c}^{0}=\mathrm{K}_{p 0} \psi \alpha \beta, \quad \Pi^{0}=\Pi_{p}^{0}+\Pi_{c}^{0}=\mathrm{K}_{0} \frac{\alpha+r \alpha^{2}}{1+\alpha} .
$$

The undimensional cutting stiffness (24) takes the following form:

$$
\kappa^{0}(\alpha ; r)=r+\frac{1-r}{(1+\alpha)^{2}}, \quad \kappa_{p}^{0}(\alpha ; r)=r_{p}+\frac{1-r_{p}}{(1+\alpha)^{2}}
$$

In the following, the zero superscript for quantities (28) will be omitted. An example of data according to (27), (28) is given on fig. 6.

It is remarkable that the cut stiffness varies in finite range.

The unperturbed motion corresponds to the cutting with constant tool deflection $\xi^{0}$, determined by equations (21), (27)

$$
\xi^{0}=\Pi^{0}
$$

For small oscillations around the reference state described by (26), (27), (29) the tool's deflection writes as follows: $\xi=\xi^{0}+\delta \xi(\tau)$. The equations (21), (23), (25) can be linearized w.r.t. the variation $\delta \xi$, yielding the variation equations of motion :

$$
\begin{aligned}
\frac{F^{2}}{4 \pi^{2}} \delta \xi(\tau)^{\prime \prime} & +2 \zeta \frac{F}{2 \pi} \delta \xi(\tau)^{\prime}+\delta \xi(\tau)= \\
& =\mathrm{K}_{p 0}\left\{\kappa_{p}\left(\alpha ; r_{p}\right)[\delta \xi(\tau-1+\beta)-\delta \xi(\tau)]+\psi[\delta \xi(\tau-\beta)-\delta \xi(\tau)]\right\}
\end{aligned}
$$

For fixed set of cut depth (parameter $B$ in equation (10)), tool and material, the quantities $\left\{\zeta, \mathrm{K}_{p 0}, r_{p}, \psi, \beta\right\}$ would be constant. Control parameters would be: cutting velocity $F$ and 

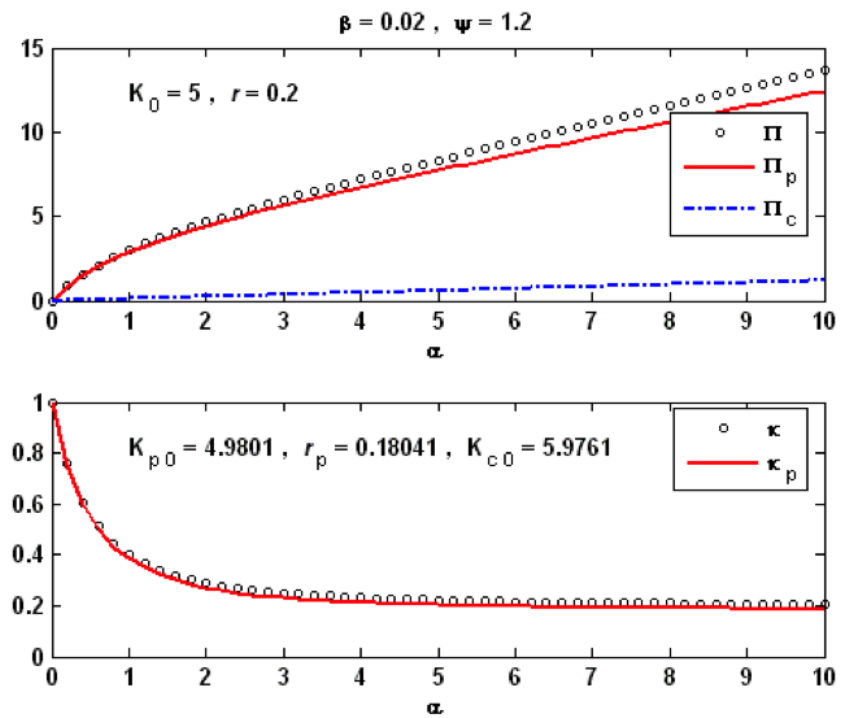

Figure 6 Quasistatic cutting forces $\Pi=\Pi_{p}+\Pi_{c}$ and cut stiffness $\kappa, \kappa_{p}$ vs feed per turn.

feed per turn $\alpha$. The parameter $\alpha$ is interrelated to $\kappa_{p}$ via equation (28), and the force value $\Pi_{p}(\alpha)$, see fig. 3, determines the tangent slope (fig. 6)

$$
\alpha=-1+\sqrt{\frac{1-r_{p}}{\kappa_{p}-r_{p}}} .
$$

Finally, the stability evaluation with ploughing taken into account, corresponds to the determination of the stability boundaries on the plane $\left\{F, \kappa_{p}\right\}$ or $\{F, \alpha\}$ according to (31) or $\{F, \kappa\}$ according to (28).

The solution of the equation (30) can be replaced by an exponential form (Euler's substitution) $\delta \xi(\tau)=\delta \xi_{0} e^{\lambda \tau}$. Thus, one obtains the characteristic equation.

$$
\frac{F^{2}}{4 \pi^{2}} \lambda^{2}+2 \zeta \frac{F}{2 \pi} \lambda+1+\mathrm{K}_{p 0}\left[\kappa_{p}\left(1-e^{-\lambda+\lambda \beta}\right)+\psi\left(1-e^{-\lambda \beta}\right)\right]=0 .
$$

If in the equation (32) one assumes $\beta=0, \mathrm{~K}_{p 0} \mapsto \mathrm{K}_{0}, \kappa_{p} \mapsto \kappa$, the resulting equation corresponds to the classical case for one cutting edge,

$$
\frac{F^{2}}{4 \pi^{2}} \lambda^{2}+2 \zeta \frac{F}{2 \pi} \lambda+1+\mathrm{K}_{0} \kappa\left(1-e^{-\lambda}\right)=0
$$

analyzed in most works on stability of continuous cutting (Altintas (2000); Tobias et al. (1958)).

The boundaries of instability zones correspond to the locus of points for which the characteristic exponent $\lambda$ is purely imaginary $-\lambda=2 \pi i s, i=\sqrt{-1}, s \in \mathbb{R}$. It is of interest to notice that in this case, the static bifurcation (Euler's bifurcation) $\lambda=0$ doesn't exist and that only the dynamic bifurcation (Poincaré - Andronov - Hopf bifurcation) is possible. 

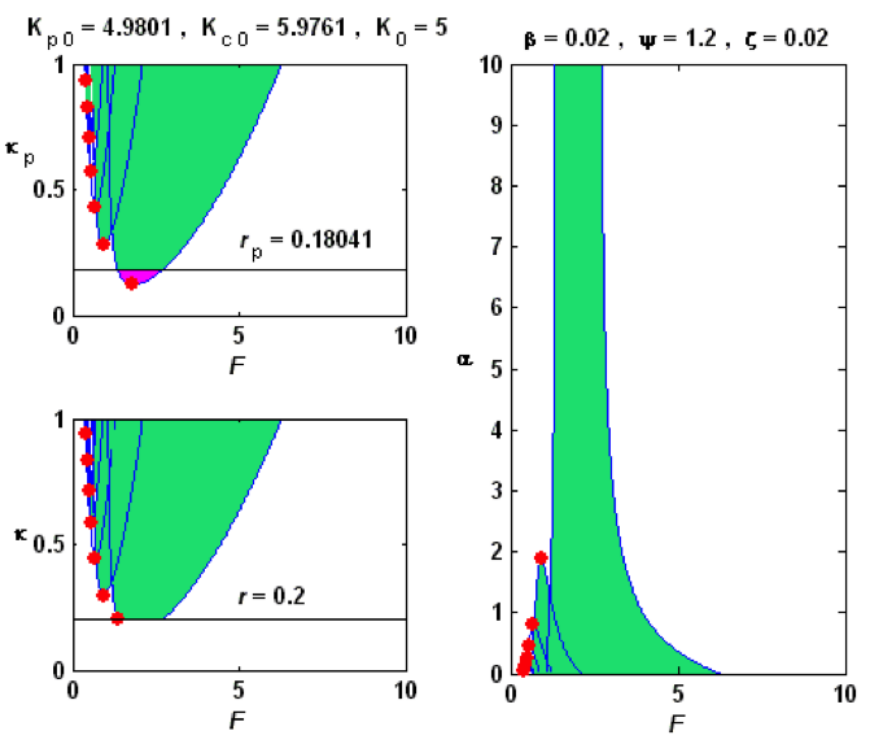

Figure 7 Instability zones for continuous cutting on parameter planes $\left\{F, \kappa_{p}\right\},\{F, \kappa\},\{F, \alpha\}$ with $\beta=0.02$.

After substitution $\lambda=2 \pi i s$ into the equation (32) and real and imaginary part separation, one obtains two transcendent equations

$$
\begin{aligned}
& -F^{2} s^{2}+1+\mathrm{K}_{p 0}\left\{\kappa_{p}[1-\cos 2 \pi s(1-\beta)]+\psi(1-\cos 2 \pi s \beta)\right\}=0, \\
& 2 \zeta F s+\mathrm{K}_{p 0}\left[\kappa_{p} \sin 2 \pi s(1-\beta)+\psi \sin 2 \pi s \beta\right]=0,
\end{aligned}
$$

which determine, in parameterized form, the values of $\left\{F, \kappa_{p}\right\}$ on the stability boundary $\left\{F(s), \kappa_{p}(s)\right\}$. The solution of the system (34) is sought under assumption $F>0, r_{p} \leq$ $\kappa_{p} \leq 1$. Depending on the values of parameters $\mathrm{K}_{p 0}, \beta, \psi, \zeta$, there would exist finite or infinite solution branches for (34). Fig. 7 shows the results of computation of stability boundaries for the case from fig. $6-\left\{\beta=0.02, \psi=1.2, \mathrm{~K}_{p 0}=4.9801\right.$ with the damping $\zeta=0.02$. It is important to remark that, for these graphs, the cut depth $B$ is taken constant. Only the chip thickness evolves with its connected quantities $\kappa, \kappa_{p}$ and $\alpha$. The areas of instability of continuous cutting are green-colored and the extrema of respective boundary curves are shown with red points.

Fig. 8 shows analogous results for $\beta=0$ with the parameter values, corresponding to fig. $7\left\{\mathrm{~K}_{p 0}, \beta, \psi, \zeta\right\}$, i.e. the ploughing effects are not considered. These plots correspond entirely to the classical results, such as shown by Altintas (2000). Nevertheless, as for the rational-fractional cutting force model, the stiffness $\kappa$ cannot be smaller than $r=0.2$ (28), a part of instability domain becomes unavailable for experimental observation. I.e. there exists a range of cutting velocity values (parameters $F$ ), in which the solution of (34) would exist for $\kappa_{p}<r_{p}$, which does not agree with (28), since $\kappa_{p}>r_{p}, \forall \alpha>0$. This means that in this range the vibrations exist for any value of feed. This fact is observable in reality but cannot be explained without a tool-workpiece interaction model with limited stiffness (fig. 6). Fig. 8 features analogous results for the case when the ploughing effect is not accounted for $(\beta=0)$. By comparing fig. 7 and 8 , we can notice that the ploughing would lead to a 

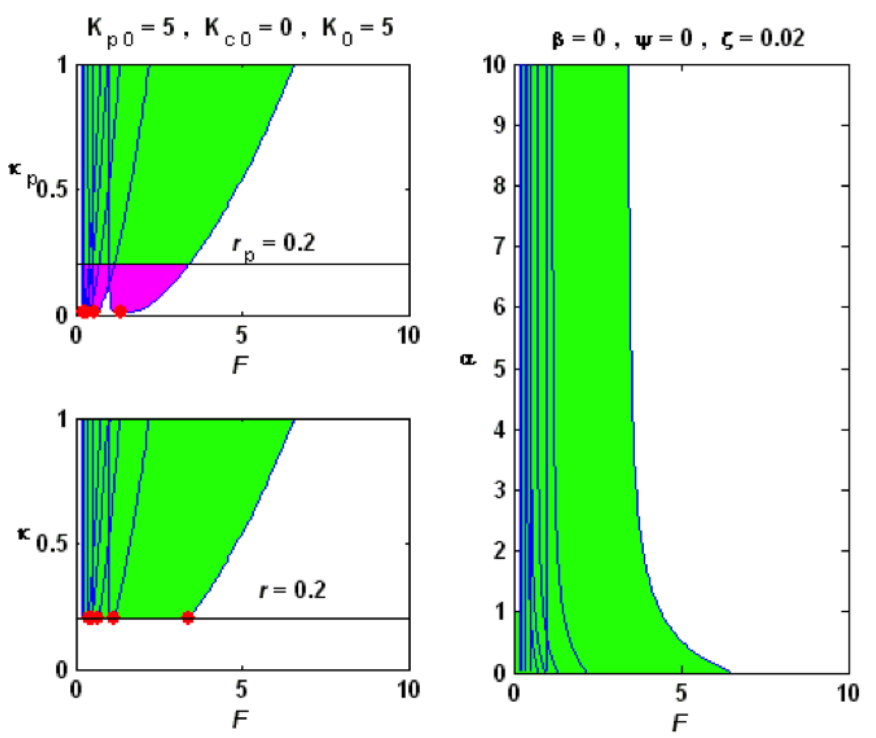

Figure 8 Areas of instability of continuous cutting in three planes of parameters $\left\{F, \kappa_{p}\right\},\{F, \kappa\}$, $\{F, \alpha\}$ at $\beta=0-$ no ploughing.

reduction of unstable areas, especially a low cutting speeds. This fact, that is observed in practice, cannot be explained in the framework of classical model.

The reduction of the cut stiffness $\mathrm{K}_{0}=K_{0} / k=\gamma \sigma_{L} B / k$ reduces the unstable areas in the plane $\left\{F, \kappa_{p}\right\},\{F, \kappa\},\{F, \alpha\}$. The results for $\mathrm{K}_{0}=0.5$ are shown on fig. 9 , this stiffness is ten times lower than in the case presented on fig. 7. The increase of the $\mathrm{K}_{0}$ enlarges instability areas. Fig. 10 shows the results for $\mathrm{K}_{0}=50$, which is ten times higher, than for the fig. 7 .

The influence of parameters $\{\psi, \beta\}$ is illustrated by the results on fig. 11, 12. Thus, the developed model allows investigation of influence of all the parameters determining cutting dynamics phenomena, including the parameters of the tool's fixation characteristics. The measurement of cutting forces in quasistatic mode allows determining the parameters $\left\{K_{0}, H, r\right\}$. The measurement of vibrations for a tool with known characteristics $\{m, k, \zeta\}$ enables the computation of stability boundaries, for example on the plane $\{F, \alpha\}$, which gives access to quantities $\{\beta, \psi\}$, characterizing ploughing.

\section{Ploughing-caused damping modification.}

Consider the case, when the flank face-related delay is relatively small $\beta \ll 1$, and apply the following approximation

$$
1-e^{-\lambda+\lambda \beta} \simeq 1-e^{-\lambda}, \quad 1-e^{-\lambda \beta} \simeq \lambda \beta, \quad \mathrm{K}_{p 0} \kappa_{p} \simeq \mathrm{K}_{0} \kappa .
$$

The equation (32) becomes analogous to the single-edge cutting (33)

$$
\frac{F^{2}}{4 \pi^{2}} \lambda^{2}+2 \zeta_{\Sigma} \frac{F}{2 \pi} \lambda+1+\mathrm{K}_{0} \kappa\left(1-e^{-\lambda}\right)=0 .
$$



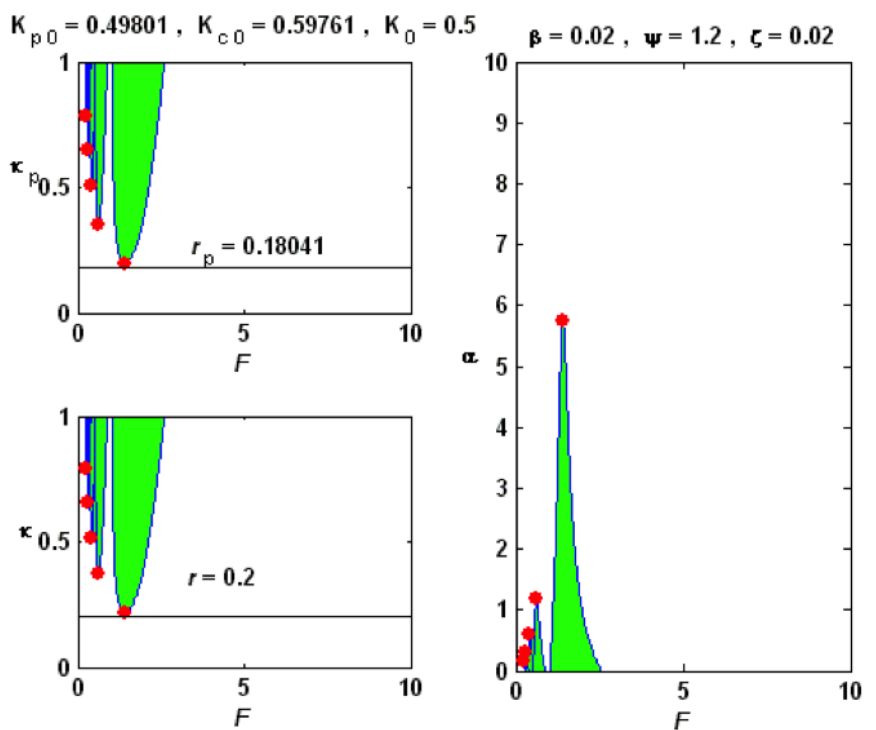

Figure 9 Reduction of instability regions. Case $\mathrm{K}_{0}=0.5$ (compare to fig. 7).
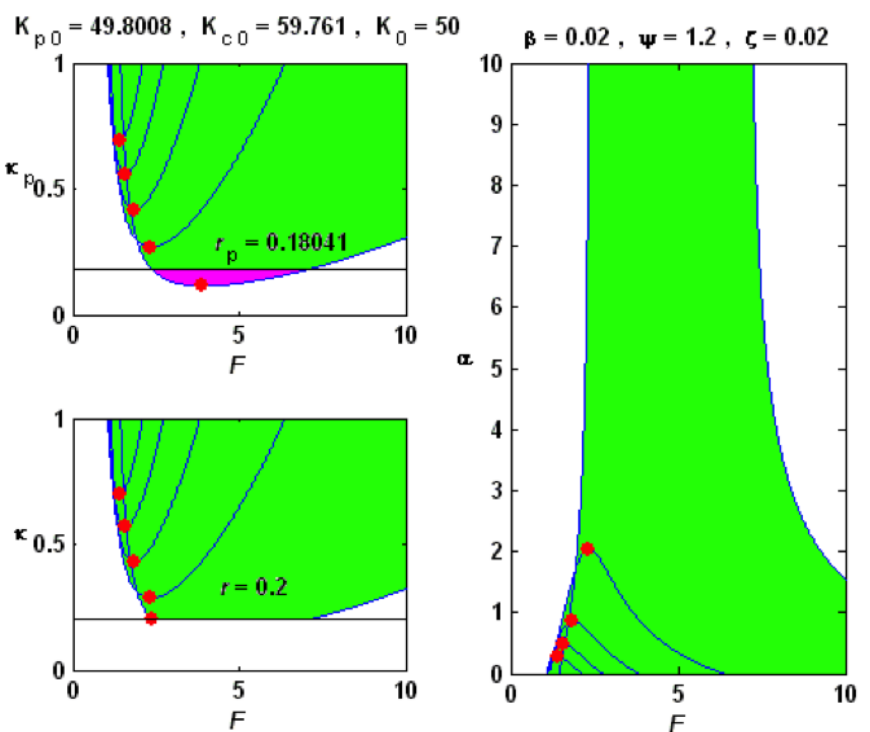

Figure 10 Enlargement of instability regions. Case $\mathrm{K}_{0}=50$ (compare to fig. 7). 

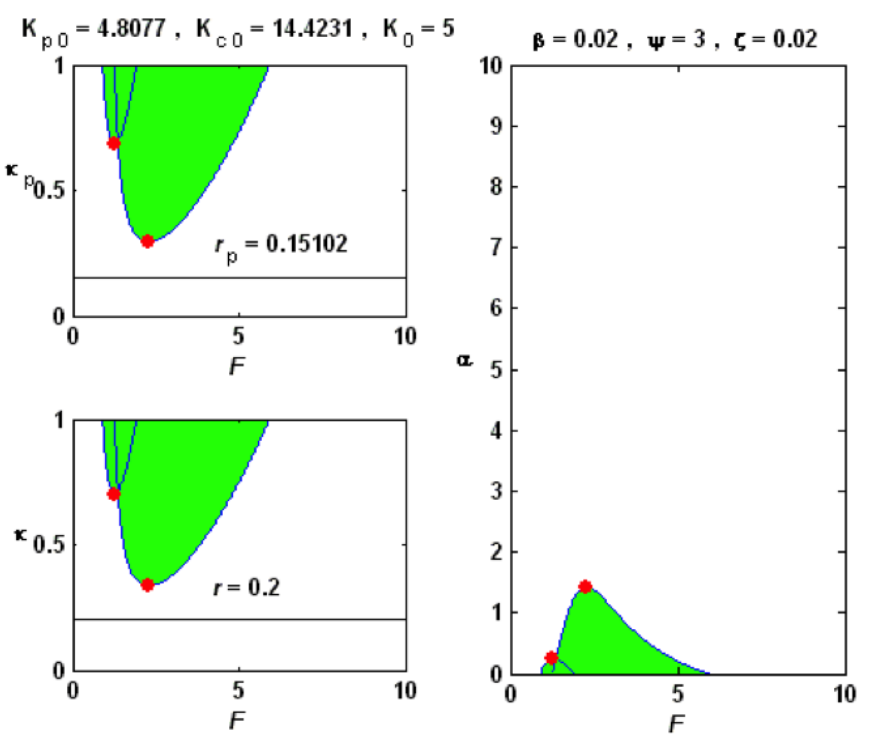

Figure 11 Raise of the instability regions on planes $\left\{F, \kappa_{p}\right\},\{F, \kappa\}$ is caused by the increase in stiffness of the flank face, $\psi=3$ (compare to fig. 7).
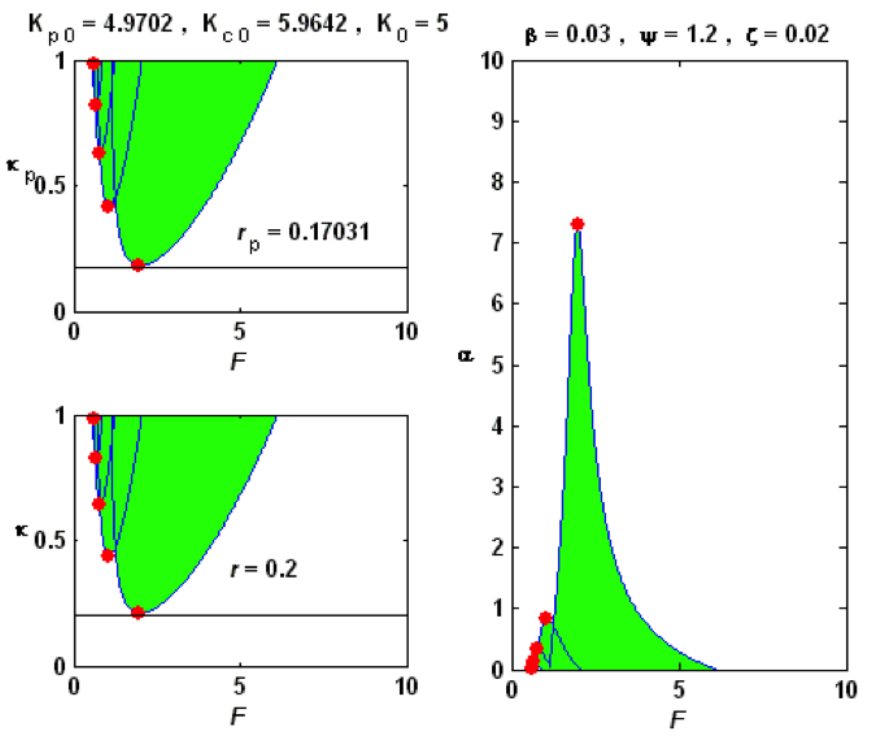

Figure 12 Deformation of instability regions, caused by the increase in $\beta=0.03$ (compare to fig. 7), i.e. in the effective auxiliary edge distance. 


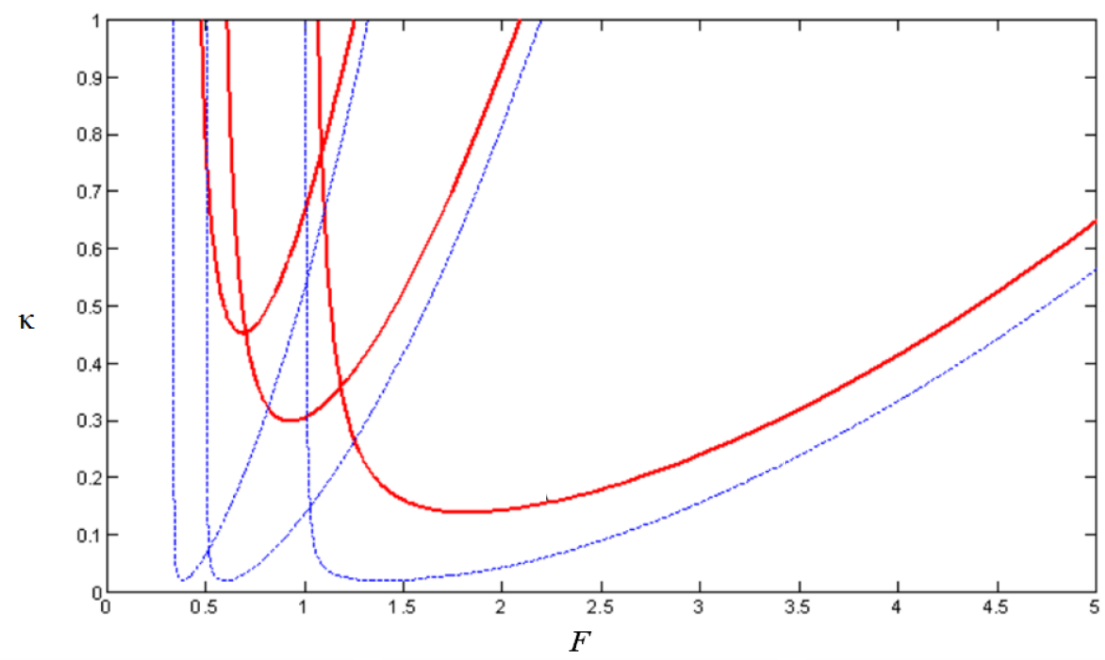

Figure 13 Stability areas for continuous cutting without ploughing (blue line) and with ploughing taken into account (red line).

The resulting overall damping coefficient $\zeta_{\Sigma}$ would consist of two terms:

$$
\zeta_{\Sigma}=\zeta+\zeta_{c}
$$

with $\zeta_{c}$ linear damping, caused by the flank face work:

$$
\zeta_{c}=\frac{\pi \mathrm{K}_{c 0} \beta}{F} \simeq \frac{\pi \mathrm{K}_{0} \psi \beta}{F}=\frac{K_{0}}{2 \sqrt{k m}} \frac{\psi b}{V_{c}} .
$$

with $V_{c}=2 \pi R / T$ - cutting velocity. The characteristic equation (35) corresponds to the variation equation (30) in the following form:

$$
\frac{F^{2}}{4 \pi^{2}} \delta \xi(\tau)^{\prime \prime}+2 \zeta_{\Sigma} \frac{F}{2 \pi} \delta \xi(\tau)^{\prime}+\delta \xi(\tau)=\mathrm{K}_{0} \kappa(\alpha ; r)[\delta \xi(\tau-1)-\delta \xi(\tau)] .
$$

Thus, the influence of the ploughing can be represented as linear damping $\zeta_{c}$ (36), proportional to the ploughing force and inversely proportional to the cutting velocity $V_{c}$. The expression of the efficient damping (36) reflects a fact well known in practice: the influence of ploughing increases at low cutting velocities (this is why in drilling, peening occurs under the center of the tool) (Tobias (1965)). The influence of ploughing on the boundaries of the stability area boundaries for turning is shown on fig. 13.

The quantity $\left(K_{c 0} b / V_{c}\right)$ is called linear cutting damping coefficient, accounting for the ploughing effect. This is consistent with the interference volume-based damping description.

\section{Conclusion}

A macroscopic mechanistic model framework for turning including ploughing effect is proposed, based on the concept of a fictitious auxiliary cutting edge, representing the 
flank face interaction. This auxiliary cutting edge is situated closely behind the principal one. The latter represents the cutting itself, as classically stipulated in literature. In this context, the tool-workpiece interaction model in this work is characterised by 5 parameters $\left\{K_{0}, H, r, b, K_{c 0}\right\}$ and describes the nonlinear dynamics of continuous and interrupted cutting. The first three cutting parameters $\left\{K_{0}, H, r\right\}$ are related to the principal edge cutting force model coefficients (the rational-fractional formulation is used in the present work). These quantities can be evaluated experimentally in the quasi-static cutting case via widespread protocols. The work of the auxiliary cutting edge is characterised by two parameters: (1) geometrical parameter $b$, related to the size of the flank face; (2) stiffness parameter $K_{c 0}$, connected to the interaction of the flank face with the machined material.

The application if the above mentioned approach to the investigated turning operation leads to the following observations:

1. The resulting dynamics involve two delays $\{T-\Delta T, \Delta T\}$. For vanishing ploughingrelated delay $\Delta T \rightarrow 0$ the model is identical to the classical model of cutting with single edge.

2. The variation of the auxiliary edge parameters $\left\{b, K_{c 0}\right\}$ has major influence on position and shape of the instability zones for continuous cutting.

3. The use of rational-fractional cutting law with finite cutting stiffness variation provides an explanation for a practically observed fact: for certain parameter combination there is a range of cutting velocities, inside which the vibrations exist at any feed per turn.

4. The additional values $\left\{b, K_{c 0}\right\}$, characterising the ploughing, can either be determined from an experiment with vibrations in the feed direction, at frequencies multiples of the workpiece rotation rate, or from experimental measurement of instability boundaries (auto-oscillation onset) for continuous cutting process.

5. An asymptotical reduction of the model leads to an analytical expression of the linear damping coefficient including the influence of ploughing. This coefficient is proportional to the flank face force interaction coefficient and inversely proportional to the cutting speed.

For further developments, an experimental investigation could constitute a natural continuation of the presented theoretical framework.

\section{Acknowledgement}

The research was partially supported via the Agreement $\mathrm{N}^{o} 14.579 .21 .0042$ 25.08.2014 (id RFMEFI57914X0042) between OAO 'VNIIINSTRUMENT' and the Ministry of Education and Science of the Russian Federation on the subject "Development of equipment and technology for nano-dimensional machining of optical materials by monocrystalline and abrasive tools under quasi-plastic cutting".

\section{References}

Altintas, Y. (2000), 'Manufacturing automation. Metal Cutting Mechanistics, Machine Tool Vibrations, and CNC Design'. Cambridge University Press. 
Bondarenko, D., Paris, H. and Gouskov, A. (2009) 'Parameters Identification on the Ploughing Force Model by an Experimental Approach', 12-th CIRP Conference on Modeling of Machining Operations, Donostia-San Sebastian, Spain, May 7-8, 2009. pp. 249-254

Bondarenko, D. (2010) 'Etude mesoscopique de l'interaction mechanique outil/piece en contribution sur le comportement dynamique du systeme usinant', PhD Thesis, INP de Grenoble \& BMSTU de Moscou. Grenoble France, 169 p.

Brissaud, D., Gouskov, A., Paris, H. and Tichkiewitch, S. (2007) 'A more realistic cutting force model at uncut chip thickness close to zero' Annals of the CIRP, Vol. 56, pp. 415-418

Fofana, M. S., Ee, K. C. and Jawahir, I. S., (2003) 'Machining stability in turning operation when cutting with a progressively worn tool insert,' Wear, Vol. 255, no. 7-12, pp. 13951403, 2003.

Enders, W.J., DeVor, R.E. and Kapoor, S.G. (1995) 'A dual-mechanism approach to the prediction of machining forces, part 1: model development' Trans ASME, Vol. 117, pp. $526-533$

Gouskov, A.M. and Voronov, S.A. (2001) 'Dynamic Models Generalization of Manufacturing Systems with Single-Point Cutting Considering Equations of New Surface Formation' Nonlinear Dynamics and Control of Mechanical Processing. Proceedings, 2nd Workshop in COST Action P4. Technical University of Budapest Department of Applied Mechanics. Hungary, June 15, 2001. pp. 7-18.

Gouskov A. M. (2013) 'Experimental determination of drilling forces based on dimensional analysis and similarity theory' (in Russian), Science and education magazine, No. 9, pp. $15-35$

Guibert, N. and Paris, H. (2008) 'Influence of the ploughing effect on the vibratory drilling behaviour' International Journal of Machining and Machinability of Materials. Vol. 3, No. 1, pp. 34Đ51

Lee, B.Y., Tarng, Y.S. and Ma, S.C. (1995) 'Modeling of the process damping in chatter vibration' Int. J. Mech. Tool Manufacturing. Vol. 35, No. 7, pp. 951-962

Mehdi, K. and Zghal, A. (2012) 'Modelling cutting force including thrust and tangential damping in peripheral milling process' International Journal of Machining and Machinability of Materials. Vol. 12, No. 3, pp. $236 Đ 251$.

Moradi, H., Bakhtiari-Nejad, F., Movahhedy, M. R. and Ahmadian, M. T. (2010), 'Nonlinear behaviour of the regenerative chatter in turning process with a worn tool: Forced oscillation and stability analysis,' Mechanism and Machine Theory, Vol. 45, No. 8, pp. 1050-1066

Paris, H., Brissaud, D., Gouskov, A., Guibert, N. and Rech J. (2008) 'Influence of the ploughing effect on the dynamic behavior of the self vibratory drilling head' Annals of CIRP - Manufacturing Technology

Seguy S. , Dessein, G. and Arnaud L. (2008) 'Surface roughness variation of thin wall milling, related to modal interactions', International Journal of Machine Tools and Manufacture, Vol. 48, No. 3-4, pp. 261-274. 
Siddhpura, M. and Paurobally, R. (2012) 'A review of chatter vibration research in turning', International Journal of Machine Tools and Manufacture, Vol. 61, pp. 27-47.

Tarng, Y. S., Young, H. T. and Lee B. Y., (1994), 'An analytical model of chatter vibration in metal cutting' , . International Journal of Machine Tools and Manufacture, Vol. 34, no. 2, pp. 183-197,

Tobias, S. A. and Fishwick, W. (1958) 'A Theory of Regenerative Chatter' Proc. IME, Vol. 170, No. 6, p. 232

Tobias, S. A. (1965) 'Machine Tool Vibration'. John Wiley and Sons, New York

Wu, D.W. (1989) 'A new approach of formulating the transfer function for dynamic cutting processes'. Journal of Manufacturing Science and Engineering, Vol. 111, No. 1, pp. $37-47$. 